\title{
Adesão dos pacientes de diabetes mellitus às atividades de autocuidado
}

\author{
Adherence of diabetes mellitus patients to self-care activities
}

João Damasceno Costa Neto1; Aldair Darlan Santos-de-Araújo²; Tatiana Cristina Fonseca Soares de Santana ${ }^{1}$, Adriana Sousa Rêgo ${ }^{1}$, Patrícia Rodrigues Ferreira ${ }^{1}$, Daniela Bassi ${ }^{1,3}$.

\begin{abstract}
Resumo: Introdução: O Diabetes Mellitus (DM) é uma doença incurável causada pela pouca ou quase nenhuma produção de insulina, onde a prevenção e o autocuidado são os caminhos para evitar complicações decorrentes de sua cronicidade. A Organização Mundial da Saúde (OMS) alerta para o autocuidado como uma medida proativa ao tratamento das doenças crônicas, por exemplo, o DM, possibilitando um estreito relacionamento entre o paciente e a doença. Objetivo: Avaliar o autocuidado nos pacientes com DM. Metodologia: Trata-se de um estudo transversal, descritivo e prospectivo realizado em uma população de 30 pacientes com DM tipo 1 e 2, com idade entre 18 e 70 anos, de ambos os sexos no Centro Especializado de Medicina - CEMESP. A adesão dos pacientes de DM as atividades de autocuidado foi avaliada por meio do "Questionário de Atividades de Autocuidado com o Diabetes" (QAD). Resultados: Foram observadas alta aderência ao autocuidado em relação ao uso das medicações e inversamente proporcional para os itens relativos às "Atividades Físicas" e "Monitorização da Glicemia". Conclusão: O resultado da amostra estudada apresentou índices de adesão ao autocuidado dentro de um quadro positivo, mas relativamente baixo e próximo da média do não desejável.
\end{abstract}

Palavras-chaves: Diabetes Mellitus; Autocuidado; Adesão.

\begin{abstract}
Introduction: Diabetes Mellitus (DM) is incurable disease caused by little or no insulin production, where prevention and self-care are the ways to avoid complications due to its chronicity. The World Health Organization (WHO) warns of self-care as a proactive measure for the treatment of chronic diseases, for example, DM, allowing a close relationship between the patient and the disease. Objective: To evaluate self-care in patients with DM. Methodology: This is a cross-sectional, descriptive and prospective study carried out in a population of 30 patients with DM type 1 and 2, aged between 18 and 70 years, of both sexes at the Specialized Medical Center - CEMESP. The adherence of DM patients self-care activities were evaluated through the "Diabetes Self-Care Activity Questionnaire" (DAQ). Results: High adherence to self-care was observed in relation to the use of medications and inversely proportional to the items related to Physical Activities and Glucose Monitoring. Conclusion: The results of the sample studied presented self-care adherence indexes within a positive but relatively low and close to the non-desirable average.
\end{abstract}

Keywords: Diabetes Mellitus; Self-Care; Accession.

\footnotetext{
'Departamento de Fisioterapia - Universidade Ceuma (UNICEUMA), São Luís - MA, Brasil 2Departamento de Fisioterapia - Centro Universitário Tiradentes (UNIT), Maceió, AL - Brasil 3Programa de Pós-Graduação em Gestão de Programas e Serviços de Saúde, Universidade Ceuma (UNICEUMA), São Luís - MA, Brasil

Departamento de Fisioterapia - Universidade Ceuma (UNICEUMA). Rua Josué Montello, Jardim Renascença II, São Luís, MA - Brasil, Telefone: +55 98 32144277, danielabassifisio@gmail.com.
} 


\section{Introdução}

O Diabetes Mellitus (DM) é um grupo de doenças metabólicas provocadas pela pouca ou quase nenhuma secreção e/ou ação de insulina, caracterizado por alterar o sistema vascular e neurológico, sendo a prevenção a forma mais eficaz de evitar a cronicidade, por se tratar de uma doença que ainda não tem cura (CHACRA, 2001). Conforme descreveu Ducam (2004), o DM corresponde ao um grave transtorno para a saúde pública, sendo considerado uma epidemia, impactando negativamente na sociedade, podendo levar o indivíduo a uma má qualidade de vida ou até a invalidez antecipada, além dos gastos econômicos pelo custeio do tratamento.

Nesse sentido, a Organização Mundial da Saúde (OMS), chama a atenção para o autocuidado como uma medida a ser adotada na prevenção e no tratamento das doenças crônicas, por exemplo, o DM, proporcionando maior vínculo entre o paciente e a doença, diminuindo assim as incapacidades que acompanham aos problemas crônicos (OMS, 2003). Comumente a hipertensão arterial sistêmica (HAS) aparece associada ao DM. Tal interação pode resultar em danos macro e microvascular capazes de desencadear alta morbidade cardiocerebrovascular, necessitando da aderência à prática de autocuidado pelo usuário, a fim de contribuir para sua integridade estrutural e funcional (SANTOS; MOREIRA 2012; MENDES et al 2015).

Com isso, Orem (2001) definiu o autocuidado como a realização de atividades em benefício próprio objetivando a manutenção da vida e o bem-estar da pessoa, relacionada a valores, regras culturais e científicas. Assim sendo, para que as medidas de adesão ao autocuidado em pacientes do DM tenham êxito se faz necessário que o mesmo disponha de comprometimento perante o tratamento referente ao uso da medicação, alimentação, não sedentarismo e modificação de costumes não compatível a sua nova rotina de vida (TIMM; RODRIGUES; MACHADO, 2013).

Quantificar o nível de adesão ao autocuidado dos pacientes com DM não é fácil, devido às terapias que envolverem as diferentes tarefas durante 0 tratamento (TOOBERT; HAMPSON; GLASGOW, 2000). Sendo que, para a avaliação da aderência ao autocuidado dos pacientes de DM ao tratamento em pesquisa, os usos de instrumentos confiáveis são validos e necessários (VICENT; McEWEN; PASVOGEL, 2008). Um recurso utilizado para avariar sistematicamente o nível de aderência no tratamento pelo autocuidado são os questionários idealizados com perguntas diretas (TOOBERT; HAMPSOM; GLASGOW, 2000). O Summary of Diabetes SelfCare Activities questionnaire (SDSCA) tem sido a ferramenta mais empregada em pesquisas deste cunho (GONZALEZ et al., 2008).

Elaborado para mensurar de forma organizada a aderência às atividades de autocuidado nos pacientes diabéticos, o mesmo encontra-se traduzido e validado no Brasil como "Questionário de Atividades de Autocuidado com o Diabetes" (QAD) (MICHELS et al., 2010).

Visto o grande aumento de complicações secundárias decorrentes do DM, se fez necessário saber como se encontra 0 autocuidado desses pacientes. Ressalta-se que uma vez que o paciente conhecendo mais sobre sua doença e sobre as consequências da falta de cuidado, ele poderá ser agente ativo em evitar problemas associados à ausência de autocuidado. 
Nesse sentido, justifica-se a realização desse trabalho. O presente estudo tem como objetivo geral verificar as atividades de autocuidado nos pacientes com DM através do questionário QAD e confirmar a hipótese que os pacientes com DM apresentam baixa aderência ao tratamento, consequentemente baixo autocuidado.

\section{Materiais e métodos}

O presente estudo é do tipo transversal, descritivo e prospectivo desenvolvido no Centro Especializado de Medicina - CEMESP, localizado na cidade de São Luís - MA, região nordeste do Brasil. O CEMESP opera como núcleo modelo no maranhão em DM e Hipertensão Arterial Sistêmica (HAS). A coleta de dados foi realizada no período de junho a setembro de 2018. Foi entrevistada uma amostra de 30 voluntários com DM.

Os critérios de inclusão foram: portadores do DM tipo 1 e 2, com idade entre 18 a 70 anos, ambos os sexos. Os critérios de exclusão foram: os incapazes de responder as perguntas, os que apresentaram sequelas decorrentes do DM (amputações de membros inferiores ou superiores e retinopatia diabética diagnosticada), desordens mentais, assim como, os que não quiseram assinar o Termo de Consentimento e Livre Esclarecido (TCLE).

Foram abordados aqueles indivíduos em ambiente hospitalar enquanto aguardavam atendimento médico ou ambulatorial, sendo a entrevista realizada em ambiente reservado e calmo.

A ferramenta utilizada para a coleta de dados foi o questionário QAD, versão traduzida, adaptada e aprovado para o Brasil do questionário SDSCA (MICHELS et tal., 2010). O QAD foi desenvolvido para mensurar de forma meticulosa a adesão às atividades de autocuidado do portador do DM. Sendo utilizado para nortear os profissionais de saúde na análise e na evolução dos pacientes. Possui 6 dimensões e 18 itens de avaliação baseados no autocuidado com o DM: "alimentação geral" (dois itens), "alimentação específica" (três itens), "atividade física" (dois itens), "monitoração da "glicemia" (dois itens), "cuidado com os pés" (três itens), "uso de medicação" (três itens) e mais três itens para avaliar o tabagismo.

Durante a coleta de dados os pacientes relataram através do questionário a periodicidade e procedimento nos últimos sete dias decorridos em uma grandeza de 0 a 7 onde o zero é um quadro menos favorável e o 7 demonstra um quadro mais positivo, excluindo os itens 2.2 e 2.3 da dimensão "alimentação específica", que pergunta a respeito da ingestão de alimentos ricos em gorduras e doces onde o padrão é inverso (se $7=0,6=1,5=2,4=3,3$ $=4,2=5,1=6,0=7$ ), como proposto no SDSCS (TOOBERT; HAMPSOM; GLASGOW, 2000). As informações sobre o consumo de tabaco foram feitas isoladamente (MICHELS et tal., 2010).

Inicialmente foi realizada uma análise descritiva. As variáveis quantitativas foram descritas por média e desvio padrão (média DP). Os dados coletados foram analisados pelo programa estatístico SPSS 18.0.

Este estudo foi aprovado pelo Comitê de Ética em Pesquisa em Seres Humanos do Centro Universitário do Maranhão - CEUMA, sob o Parecer $\mathrm{n}^{\circ}$ 2.469.206.

\section{Resultados}


A tabela 1 descreve os dados referentes à caracterização sociodemográficas.

A tabela 2 descreve os dados das variáveis clínicas da população estudada.

A Tabela 3 descreve os resultados obtidos através das dimensões e itens do QAD.

Para a melhor compreensão dos resultados apurados, as médias encontradas de 0 a 3 dias por semana serão consideradas como um quadro ruim ou menos favorável e com baixo índice de adesão dos pacientes de DM às atividades de autocuidado. As médias entre 4 e 7 dias por semana, serão aceitas como boas e dentro de um quadro positivo, com exceção dos itens 2.2 e 2.3 da dimensão "alimentação específica". Conforme os resultados apresentados por Visentin et al. (2016), fazendo uso da ferramenta QAD, no estudo do Autocuidado de usuários com diabetes tipo 1.

Tabela 1. Caracterização das variáveis sociodemográficas da população estudada.

\begin{tabular}{llll}
\hline Variáveis (n=30) & Categoria & $\mathbf{N}$ & $(\%)$ \\
\hline Sexo & Feminino & 25 & $(83,3)$ \\
& Masculino & 05 & $(16,7)$ \\
Faixa Etária (anos) & & & \\
& $18-34$ & 05 & $(16,7)$ \\
& $35-49$ & 06 & $(20,0)$ \\
& $50-59$ & 10 & $(33,3)$ \\
Raça & $60-70$ & 09 & $(30,0)$ \\
& & & \\
& Parda & 16 & $(53,3)$ \\
Estado Civil & Branca & 11 & $(36,7)$ \\
& Negra & 03 & $(10,0)$ \\
& Casado (a) & 21 & $(70,0)$ \\
Escolaridade & Solteiro (a) & 05 & $(16,7)$ \\
& Viúvo (a) & 04 & $(13,3)$ \\
& & & \\
& Superior & 06 & $(20,0)$ \\
& Ensino Médio Completo & 04 & $(13,3)$ \\
Profissão & Ensino Médio Incompleto & 03 & $(10,0)$ \\
& Fundamental completo & 06 & $(20,0)$ \\
& Fundamental Incompleto & 11 & $(36,7)$ \\
& & & $(43,3)$ \\
& Empregado & 13 & $(36,7)$ \\
Total & Aposentado & 11 & $(6,3)$ \\
\hline & Desempregado & 04 & $(\mathbf{1 0 0})$ \\
\hline
\end{tabular}


Tabela 2. Caracterização das variáveis clínicas da população estudada.

\begin{tabular}{|c|c|c|c|}
\hline Variáveis $(n=30)$ & Categoria & $\mathbf{N}$ & (\%) \\
\hline +DM Tipo 1 & & 06 & (20) \\
\hline +DM Tipo 2 & & 24 & (80) \\
\hline \multirow[t]{3}{*}{ Tempo DM (anos) } & $0-10$ & 24 & (80) \\
\hline & $11-20$ & 04 & $(13,33)$ \\
\hline & $21-30$ & 02 & $(6,66)$ \\
\hline "HAS & & 14 & $(46,66)$ \\
\hline Sem *HAS & & 16 & $(53,33)$ \\
\hline \multirow[t]{2}{*}{ Tempo de *HAS (anos) } & $0-10$ & 10 & $(71,42)$ \\
\hline & $11-20$ & 04 & $(28,57)$ \\
\hline \multirow[t]{4}{*}{ §IMC (Kg/m²) } & Normal & 10 & $(33,33)$ \\
\hline & Sobre Peso & 15 & (50) \\
\hline & Obesidade Grau 1 & 03 & (10) \\
\hline & Obesidade Grau 2 & 02 & $(6,33)$ \\
\hline Sedentário & & 21 & (70) \\
\hline Ativos & & 09 & (30) \\
\hline Total & & 30 & $(100)$ \\
\hline
\end{tabular}

*DM: diabetes mellitus; $\quad$ +HAS: Hipertensão Arterial Sistêmica; §IMC: Índice de Massa Corpórea. 
Tabela 3. Itens do questionário de Autocuidado com o Diabetes

\begin{tabular}{lc}
\hline \multicolumn{1}{c}{ Itens do QAD } & Média ('DP) \\
\hline Seguir uma dieta saudável & $4,48(3,10)$ \\
Seguir uma orientação alimentar & $4,19(2,52)$ \\
Ingerir cinco ou mais porções de frutas ou vegetais & $5,06(2,02)$ \\
Ingerir alimentos ricos em gorduras & $2,44(2,72)$ \\
Ingerir doces & $1,48(1,20)$ \\
Realizar atividade física por pelo menos 30 minutos diários & $1,87(2,29)$ \\
Realizar exercício físico específico (caminhar, nadar, etc.) & $1,73(2,41)$ \\
Avaliar o açúcar no sangue & $2,07(2,25)$ \\
Avaliar o açúcar no sangue o número de vezes recomendado & $1,70(2,62)$ \\
Examinar os pés & $5,45(2,33)$ \\
Examinar dentro dos sapatos antes de calçá-los & $4,55(3,23)$ \\
Secar os espaços entre os dedos dos pés depois de lavá-los & $4,92(2,72)$ \\
Tomar seus medicamentos conforme recomendado & $6,50(1,41)$ \\
Tomar injeção de insulina conforme recomendado & $6,33(1,64)$ \\
Tomar número indicado de comprimido de diabetes & $6,20(1,87)$ \\
\hline
\end{tabular}

*DP (Desvio Padrão).

\section{Discussão}

Os principais achados deste estudo foram: i) no perfil sociodemográficos da amostra investigada, verifica-se maior regularidade de participantes do sexo feminino e casados (as), com idade média entre 50 a 60 anos, com baixa escolaridade e a maioria declarou ter renda mensal. ii) analisando os dados clínicos, observa-se que a maioria dos pacientes são classificados com IMC no sobrepeso e o tempo médio de diagnóstico relatado por um médico para DM e HAS foi de 0 a 10 anos. iii) as dimensões que mais afastam os pacientes de DM das atividades de autocuidado foram "atividade física" e "monitorização da glicemia", não afetando o contexto geral de um bom autocuidado.

Neste estudo o baixo nível de escolaridade dos integrantes pode prejudicar de forma considerável na participação dos pacientes ao tratamento do DM, o que pode ocorrer a falta de compreensão na dinâmica da doença por parte do usuário, estabelecendo assim um maior desafio para a equipe multidisciplinar (TORRES, 2010). Há uma relação direta entre 0 baixo nível de autocuidado e o baixo índice escolar, ou seja, quanto menos escolaridade possuir a pessoa, menor será seu autocuidado (BARBUI, 2002).

$\mathrm{Na}$ faixa etária da população estudada nesta pesquisa, observou-se a maior frequência dos participantes 
com idade acima dos 50 anos, concordando com Visentin et al. (2016), no seu estudo em autocuidado de usuários com diabetes tipo 1 utilizando o QAD. Além disso, $46,66 \%$ dos indivíduos deste estudo apresentam associada ao DM a HAS, podendo culminar em maiores riscos de doenças cardiovasculares e renais. No entanto, a literatura é assertiva em afirmar que um controle rigoroso da HAS proporciona benefícios em pacientes portadores dessas patologias, com redução significativa dos riscos apresentados em todos os pontos associados ao DM (LASTRA et al, 2014).

Em relação ao sexo, neste estudo houve maior presença das mulheres, que segundo Zaitune et al. (2006), no estudo com 426 pacientes com idade maior que 60 anos, certificaram que o sexo feminino tem maior percepção sobre a doença do que o sexo masculino, além denotar melhor entendimento das patologias apropriados ao autocuidado.

A correlação entre o nível de instrução e adesão às atividades de autocuidado foi negativa, negativa entre instrução e monitorização da glicêmica, positiva entre sexo e o cuidado com os pés, positiva entre idade e medicação e positiva entre renda e alimentação geral e específica.

Neste estudo os resultados referentes à dimensão "atividade física" obteve a mais baixa adesão. No item "Realizar atividade física por pelo menos 30 minutos", média de 1,87 dias por semana, e no item "realizar exercício físico específico (caminhar, nadar etc.)", média de 1,73 dias por semana. Este resultado corrobora com Silva (2016), na pesquisa com 360 usuários, para conhecer as atividades de autocuidado em DM tipo 2: fatores associados, realizado no Hospital Universitário em São Luís capital do Maranhão, utilizando a mesma ferramenta para a coleta de dados. No item "realizar atividade física por pelo menos 30 minutos", média de 2,81 dias por semana e no item "realizar exercício físico específico (caminhar, nadar etc.)", média de 1,60 dias por semana. Os baixos índices em ambos os estudos devem estar relacionados às peculiaridades sociodemográficas das populações entrevistadas. Utilizando o mesmo questionário (QAD) em sua pesquisa sobre a relação entre o tempo e o diagnóstico de DM tipo II e o autocuidado, Santos (2013) discorda deste estudo ao encontrar a média de 6,8 dias por semanas somente no item "realizar atividade física por pelo menos 30 minutos diários". Este alto índice poderia ser comprovado devido às atividades realizadas diariamente arrumação e limpeza da casa, passeios, saídas, compras e trabalho e etc.

Neste estudo, quando perguntado sobre a "monitorização da glicemia", os resultados mostram baixa aderência. No item "avaliar o açúcar no sangue" média de 2,07 dias por semana, e no item "avaliar o açúcar no sangue o número de vezes recomendado", média de 1,70 dias por semana. Dados parecidos encontramos na amostra que utilizou O QAD como instrumento de pesquisa em 35 pessoas para identificar adesão ao tratamento de pacientes com DM tipo 2, realizada por Ross (2015). No item "avaliar o açúcar no sangue" média de 3,60 dias por semana, e no item "avaliar o açúcar no sangue 0 número de vezes recomendado", média de 2,29 dias por semana. Em ambos os estudos a amostragem era não probabilística e por conveniência e os participantes não estavam escritos em nenhum programa de controle de diabetes. As médias baixas nesta dimensão pode ser compreendida, por ser uma atividade inconveniente, por falta do monitorador glicêmico pessoal ou pela ausência de 
entendimento da magnitude do controle da doença (ROOS, 2015). Divergindo destes estudos encontramos Gomides et al. (2013) pesquisando o autocuidado das pessoas com diabetes mellitus que possuem complicações em membros inferiores através da ferramenta $Q A D$, obtendo a média no item "avaliar o açúcar no sangue" de 5,8 dias por semana e no item "avaliar o açúcar no sangue o número de vezes recomendado" de 4,3 dias por semana. Todos os pacientes que fizeram parte do referido estudo já possuíam alguma complicação nos membros inferiores, critério de inclusão do estudo, e frequentemente utilizavam os ambulatórios para realização de curativos e verificação glicêmica, sendo a causa provável das altas médias.

O consumo de tabaco não foi analisado como causador da não adesão às atividades do autocuidado, mas sim como uma característica comportamental do paciente. O QAD apresenta somente as alternativas de resposta, "fumante" ou "não fumante".

Nesta dimensão $66,66 \%$ dos entrevistados nunca tiveram hábitos tabágicos, ficando bem aproximado aos valores encontrados por Silva (2016) no estudo com 360 usuários para conhecer as atividades de autocuidado em DM tipo 2: fatores associados, que representou $66,94 \%$ de não fumantes.

O consumo do tabaco é um fator desencadeante de uma sequência de patologias crônicas, particularmente as doenças pulmonares, cardiovasculares e o câncer, sendo o principal responsável mundialmente por causar mortes que poderiam ser evitadas (WHO, 2011).

\section{Conclusão}

Os indivíduos que participaram deste estudo apresentaram um bom nível de autocuidado. As dimensões que demandam mais vigilância ("monitorização da glicemia" e "cuidado com os pés") e as que requerem uma mudança no estilo de vida ("atividade física" e "alimentação"), ainda precisam ser mais trabalhadas. Neste sentido é recomendado que haja um alerta para a situação sobre a importância do autocuidado. No presente estudo a maior limitação se deu por parte dos participantes em não se recordarem dos procedimentos das atividades do autocuidado nos últimos sete dias decorridos. 


\section{Referências}

Barbui EC, Cocco MI. Conhecimento do cliente diabético em relação aos cuidados com os pés. Revista da Escola de Enfermagem da USP [Internet]. 2002 [acessado em 2018 nov 8]; 36(1): 97-103. Disponível em: http://www.scielo.br/scielo.php?script=sci a bstract\&pid=S0080$62342002000100014 \& \operatorname{lng}=$ pt\&nrm=iso\&tlng =en.

Chacra AR. Diabetes mellitus. IN: Prado FC, Ramos JÁ, Borges DR, Rothschild HA, eds. Artes Médicas. Tratado de Atualização Terapêutica. 20a ed. São Paulo: Câmara Publicadora do Livro, 2001, p.375-89.

Ducan BB, Schmidt MI, Giugliani ERJ. Medicina Ambulatorial: Condutas de Atenção Primária Baseadas em Evidências. 3. ed. Porto Alegre: Artmed; 2004.

Gomides DS, Villas-Boas LCG, Coelho ACM, Pace AE. Autocuidado das pessoas com diabetes mellitus que possuem complicações em membros inferiores. Acta Paulista de Enfermagem [Internet]. 2013 [acessado em 2018 nov 8]; 26(3): 289-93. Disponível em: http://www.scielo.br/scielo.php?pid=S010321002013000300014\&script=sci abstract\&t lng=pt.

Gonzalez JS, Delahanthy LM, Safren SA. Differentiating symptoms of depression from diabetes-specific distress: relationships with self-care in type 2 diabetes. Diabetologia [Internet]. 2008 [acessado em 2018 nov 8]; (51)10: 1822-5. Disponível em: https://www.ncbi.nlm.nih.gov/pmc/articles/P MC2678064/.

Lastra G, Syed S, Kurukulasuriya LR, Manrique C, Sowers JR. Type 2 diabetes mellitus and hypertension: An update. Endocrinology and Metabolism Clinics of North America [Internet]. 2014 [acessado em 2018 dec 6]; 43(1): 103-122. Disponível em: https://www.ncbi.nlm.nih.gov/pmc/articles/P MC3942662/.

Mendes CRS, Souza TLV, Felipe GF, Lima FET, Miranda MDC. Comparação do autocuidado entre usuários com hipertensão de serviços da atenção à saúde primária e secundária. Acta Paulista de Enfermagem [Internet]. 2015 [acessado em 2018 dec 6]; 28(6): 580-586. Disponível em: http://www.scielo.br/pdf/ape/v28n6/1982-

0194-ape-28-06-0580.pdf.

Michel MJ, Coral MH, Sakae TM, Damas TB. Questionário de atividades de autocuidado com diabetes: tradução, adaptação e avaliação das propriedades psicométricas. Arquivos Brasileiros de Endocrinologia e Metabologia [Internet]. 2010 [acessado em 2018 nov 8]; 54(7): 644-50. Disponível em: http://www.scielo.br/scielo.php?script=sci a rttext\&pid=S0004-27302010000700009.

Orem DE. Nursing: Concepts of Practice. 6th ed. St Louis (USA): Mosby; 2001.

ORGANIZAÇÃO MUNDIAL DA SAÚDE - OMS. Cuidados inovadores para condições crônicas: componentes estruturais de ação: relatório mundial. Brasília (DF): OMS; 2003.

Ross AC, Baptista DR, Miranda RC. Adesão ao tratamento de pacientes com Diabetes Mellitus tipo 2. Demetra. 2015; 10(2): 329346.

Santos AM, Paixão CAP, Santiago MAMT. Relação entre o tempo de diagnóstico de Diabetes Mellitus Tipo II e o autocuidado dos pacientes de um município de Minas Gerais [monografia]. Minas Gerais; 2013.

Santos JC, Moreira TMM. Fatores de risco e complicações em hipertensos/diabéticos de uma regional sanitária do nordeste brasileiro. Revista da Escola de Enfermagem da USP [Internet]. 2012 [acessado em 2018 dec 6]; 46(5): 1125$1132 . \quad$ Disponível em: http://www.scielo.br/pdf/reeusp/v46n5/13.pd f.

Silva CG. Conhecimento e atividades de autocuidado dos portadores de DM tipo 2: fatores associados (dissertação). São Luís: Universidade Federal do Maranhão; 2016.

Timm M, Rodrigues MCS, Machado VB. Adherence to treatment of type 2 diabetes mellitus: a systematic review of randomized clinical essays. Journal of Nursing [Internet]. 2013 [acessado em 2018 dec 5]; 7(4): 12041215. Disponível em: https://periodicos.ufpe.br/revistas/revistaenf ermagem/article/view/11599.

Torres HC, Pace AE, Stradioto MA. Análise sociodemográfica e clínica de indivíduos com diabetes tipo 2 e sua relação com o autocuidado. Cogitare Enfermagem 
[Internet]. 2010 [acessado em 2018 dec 5]; 45(5): 1077-1082. Disponível em: https://revistas.ufpr.br/cogitare/article/viewF ile/17144/11286.

Toobert DJ, Hampson SE, Glasgow RE. The summary of diabetes self-care activities measure: results from 7 studies and a revised scale. Diabetes Care [Internet]. 2010 [acessado em 2018 dec 5]; 54(7): 644-650. Disponível em: https://www.ncbi.nlm.nih.gov/pubmed/1089 $\underline{5844}$.

Vincent D, Mcewen MM, Pasvogel A. The validity and reliability of a Spanish version of the summary of diabetes self-care activities questionnaire. Nursing Research [Internet]. 2008 [acessado em 2018 dec 5]; 57(2): 101106. Disponível em: https://www.ncbi.nlm.nih.gov/pubmed/1834 $\underline{7481}$.

Visetin A, Mantovani MF, Caveião C, Hey AP, Scheneider EP, Paulino V. Autocuidado de usuários com diabetes tipo $1 \mathrm{em}$ uma unidade básica de saúde. Revista de Enfermagem UFPE online [Internet]. 2016 [acessado em 2018 dec 5]; 10(3): 991-998. Disponível

em: http://pesquisa.bvsalud.org/ses/resource/pt/ bde-29615.
WORLD HEALTH ORGANIZATION (WHO). WHO report on the global tobacco epidemic, 2011: warning about the dangers of tobacco. Geneva: WHO, 2011.

Zaitune MPA, Barros MBA, César CLG, Corandina L, Goldbaum M. Hipertensão Arterial em idosos: prevalência, fatores associados e práticas de controle no município de Campinas, São Paulo, Brasil. Cadernos de Saúde Pública [Internet]. 2006 [acessado em 2018 dec 5]; 22(2): 285-294. Disponível

em: http://www.scielo.br/scielo.php?pid=S0102311X2006000200006\&script=sci abstract\& tlng=pt. 